\section{ESA Selects INTEGRAL}

The Science Programme Committee of the European Space Agency (ESA) formally approved in early June a further "MediumSized" scientific mission for ESA. The selection took place at the end of the Phase A study of four competing missions. Besides INTEGRAL, there were MARSNET (surface and atmospheric properties of Mars), PRISMA (astroseismology and stellar activity), and STEP (measurement of the weak equivalence principle)

Formal selection followed the recommendation, in late April, of the advisory bodies of the ESA Science Programme formulated after a public presentation of the missions and discussions, first in specialised committees and finally in a multidisciplinary body, the Space Science Advisory Committee.

The Phase A studies covered scientific goals, instruments and the satellite, and programmatic aspects, including (in the case of STEP, MARSNET and INTEGRAL) the share of activities between the partners. It was imperative that the cost to ESA of the mission be within an envelope of $322 \mathrm{MAU}$. All these aspects were considered in the presentation of the missions and in subsequent discussions.

Studied in collaboration with NASA and the Institute of Space Research, Moscow, INTEGRAL will observe cosmic gamma rays up to $10 \mathrm{MeV}$ with a spatial resolution of about 17 arcmin and a capability to locate compact sources to within 1 arcmin; the spectral resolution will be about 500 at 1 $\mathrm{MeV}$. These properties are needed to study regions such as the centre of our galaxy where a number of sources are located close to one another, and where narrow and broad emission lines of electron and positron annihilation have been observed. Understanding this complex region requires the ability to observe sources individually and to study line profiles.

Goals will be met with two instruments: an imager made of 2500 segmented CsI bars, each corresponding to a pixel of the gammaray image, and a spectrometer made of nine, cooled Ge crystals. Both will be equipped with a coded mask $4 \mathrm{~m}$ above the detectors to provide imaging. There will be two smaller instruments, namely an x-ray monitor to extend the energy range and an optical transient camera to attempt to localize possible optical counterparts to the elusive gammaray bursts.

Launching is planned for 2001 and INTEGRAL will operate like an observatory, with most of the time available to the astronomical community through peer reviewed proposals for specific observations; Science Data Centres in Europe and in the US will distribute data and services. Selection of an ESA mission means that the budget for the complete mission including two years of operations is approved. This gives a large stability to the ESA Programme. Although decisions can only be made based on the information available, the real value of any decision depends mostly on what is done with it. The selection of INTEGRAL gives astronomers the responsibility to make the best use of the opportunity given to them.

T. Courvoisier, Geneva Observatory

\section{Few Changes to HCM}

The Commission of the EC aims to seek adoption by the EC Council of Ministers this autumn of its proposed 4th Framework programme 1994-98 (F4) for science and technology; proposals for individual programmes to be implemented by the end of 1994 and overlapping with the 3rd Framework 1990-94 (F3) would follow. F4 has as its perspective the Maastricht treaty mandating the CEC to treat socio-economic areas so new programmes for transport and socio-economic research complement the F3 R\&D programmes. A. Ruberti, the EC Commissioner for science, indicated to Council in April that he will seek 13100 MECU compared with the 6600 MECU budgeted for F3. Some 790 MECU is assigned for cooperation with international organizations and non-EC countries, together with $600 \mathrm{MECU}$ for disseminating results; Human Capital and Mobility (HCM) is slated to receive $785 \mathrm{MECU}$ as compared with 587 MECU for F3.

It seems that Commissioner Ruberti wil not seek a mandate to short cut the so-called written procedure which began to be applied a few years ago to ensure full accountability and is responsible for long delays in announcing the decisions of selection rounds.

Regarding $\mathrm{HCM}$ activities in $\mathrm{F} 4$, the programme management committee (called the Committee of Regulatory Nature) representing Member States (MS) will have decisionmaking powers and not simply a consultative rôle as in F3. So it will be strengthened with respect to the CODEST committee, which in the pre-HCM SCIENCE Plan made selection decisions but presently organizes peer reviews. HCM will probably not be decentralised to MS to handle problems such as variations in the treatment of fellows between different countries. This could represent "renationalisation" of funds which should be spent for the benefit of the entire Community; the principle of the advanced control of spending may also be jeopardised. Unlike the case for F3, major shifts in $\mathrm{HCM}$ activities and decision-making processes are not on the cards so Council will probably not delay implementation of $\mathrm{HCM}$ once $\mathrm{F} 4$ is approved. It seems the CEC's proposals for HCM could imply:

- ending the institutional fellowships (applications are made to preselected institutes) because the two-step procedure is too long;

- reverting back towards the twinning arrangements used in SCIENCE Plan;

- more-or-less the same support for user access to large-scale facilities, but the inclusion of support for technical development (F3 only allows equipment expenses);

- having Euroconferences in either accessory measures or individual fellowships.

Regarding collaboration with international organizations, the problem has been that MS will not pay twice (once within the EC context and again as a member of the organization). The CEC, on the other hand, does not want to simply hand over funds. The result is that some major international facilities are not used to the full. Organizations such as CERN are therefore negotiating Cooperation Agreements with the EC which stress user access.

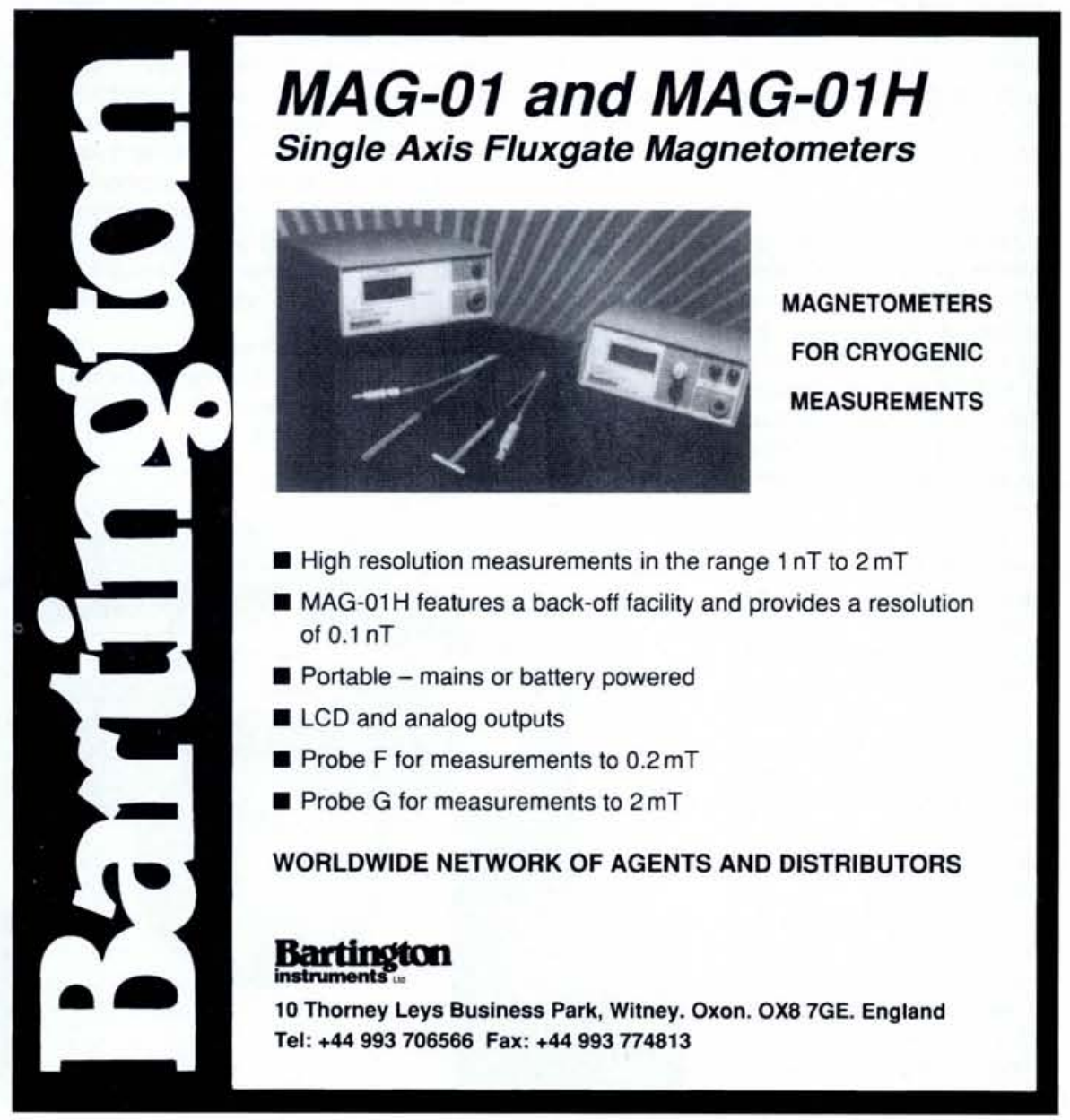

